ENCYCLOPÉDIE Encyclopédie berbère

BERBERE

32 | 2010

32 | Mgild - Mzab

\title{
Mzab - Mozabite : Langue et littérature
}

\section{S. Chaker, S. Chemakh et A. Nouh}

\section{OpenEdition}

Journals

Édition électronique

URL : https://journals.openedition.org/encyclopedieberbere/678

DOI : 10.4000/encyclopedieberbere.678

ISSN : 2262-7197

\section{Éditeur}

Peeters Publishers

\section{Édition imprimée}

Date de publication : 31 décembre 2010

Pagination : $5181-5190$

ISBN : 978-90-429-2369-0

ISSN : 1015-7344

\section{Référence électronique}

S. Chaker, S. Chemakh et A. Nouh, « Mzab - Mozabite : Langue et littérature », Encyclopédie berbère [En ligne], 32 | 2010, document M154, mis en ligne le 11 novembre 2020, consulté le 17 février 2022. URL : http://journals.openedition.org/encyclopedieberbere/678; DOI : https://doi.org/10.4000/ encyclopedieberbere.678

Ce document a été généré automatiquement le 17 février 2022.

(ㄷ) Tous droits réservés 


\title{
Mzab - Mozabite : Langue et littérature
}

\author{
S. Chaker, S. Chemakh et A. Nouh
}

[Nous remercions K. Naït-Zerrad (INALCO) pour les données chiffrées et les données linguistiques qu'il nous a fournies.]

1 Le dialecte mozabite ou tumzabt présente quasiment toutes les caractéristiques des parlers dits zénètes*. Déjà dans sa vieille classification, présentée comme provisoire, $\mathrm{R}$. Basset (1887: 3) met le mozabite dans la famille des dialectes qu'il appelle "intermédiaires », et qui sont pratiquement tous zénètes. Même si tous les auteurs n'emploient pas la dénomination « zénète » et même si les regroupements opérés ne se recouvrent pas totalement et peuvent même présenter de sérieuses divergences, les travaux récents consacrés à (ou abordant) la question de la classification des dialectes berbères tendent tous à confirmer la réalité d'un ensemble linguistique constituant une large bande (maintenant) discontinue Sud-Est/Nord-Ouest, allant de la Libye au Maroc oriental et incluant le nefousi*, les parlers de Djerba*, le ouargli*, le chaoui*, les parlers berbères du Sahara (à l'exception du touareg*, sauf pour Willms), les parlers du Centre et de l'Ouest algérien, le rifain*... Et l'appartenance du mozabite à cet ensemble est admise par tous (cf. Willms 1980, Kossmann 1999 et surtout l'essai le plus récent et le plus convaincant en la matière : Naït-Zerrad 2002).

2 La variété berbère mozabite reste encore de nos jours peu connue en raison du très petit nombre d'études qui lui ont été consacrées. En dehors de quelques travaux d'ethnologie et de présentations, très vieillies, de la grammaire mozabite datant de la fin du XIXe siècle (Moulieras 1895, Ben Lounis 1897 ; Gourliau 1898), les études de Jean Delheure constituent l'essentiel des connaissances sérieuses sur le système linguistique mozabite. Dans son «Etude sur le mozabite " (1989) qu'il présente comme un guide pour son Dictionnaire Mozabite-français (1984), et dans ses travaux antérieurs, Delheure donne des indications détaillées sur la phonologie et le système morphosyntaxique du mozabite.

3 A la lumière de ces travaux, les principaux traits linguistiques du mozabite sont les suivants (tous les exemples proviennent de Delheure 1986/1989) : 


\section{Phonologie}

4 - Le système vocalique est de type " classique ", avec trois voyelles phonologiques (/i, a, $\mathrm{u} /$ ), sans distinction de durée, et un schwa ([ə]) purement phonétique, servant d'appui syllabique; le mozabite ne présente donc apparemment aucune spécificité par rapport à la phonologie des grands dialectes berbères Nord.

5 - Au plan phonétique, on peut noter une tendance forte, peut-être plus marquée que dans d'autres dialectes, à l'ouverture de la vélaire $/ \mathrm{u} /(>[\mathrm{o}])$ et de la palatale /i/ > [e], en particulier en contexte pharyngalisé ou vélaire, ce qui est classique, mais aussi à la finale absolue : buḍ, « fond » (> [bōd]], tițt, « œil » [> [tēțț], yě̌žu > [yežžō], « il a laissé ».

6 - Le système consonantique présente une tendance forte à traiter les palato-vélaires /

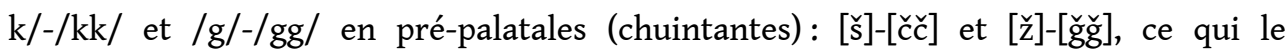
distingue nettement du kabyle par exemple (Nouh 2008) :

aždid < agḍị, « oiseau », ažužil < agujil, « orphelin », arğaz < argaz « homme »; tišli <

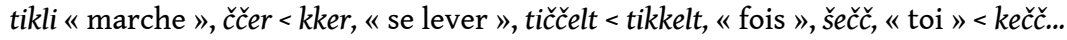

7 - Absence de spirantisation et d'affriction des dentales, contrairement à la plupart des parlers de l'Algérie du Nord, comme le chaoui (partiellement), le kabyle ou les parlers de l'Algérie centrale et occidentale..

8 - La labiovélarisation est très rare; elle est relevée seulement pour les palato-vélaires sonore $\left[\mathrm{g}^{\mathrm{w}}\right]$ et sourde $\left[\mathrm{k}^{\mathrm{w}}\right]$ : aseg ${ }^{w}$ as, « année ", yewwakw , " mon frère », regg ${ }^{w} e l$, « fuir " (aoriste intensif).

9 - A la limite entre phonologie et morphologie, la tendance à la chute de la voyelle initiale du nom, dans certains contextes phonologiques (noms de forme (v) CvC...), caractéristique des parlers zénètes, y est bien représentée :

dar < adar, "pied", fus < afus, "main », lum < alum, "paille», fiyer < ifizer,

« serpent».

\section{Morphosyntaxe}

10 - La mozabite a conservé l'intégralité de l'ancienne numération berbère, même si dans l'usage on a tendance à utiliser la numération arabe à partir de 21 .

11 - Le déictique de proximité (suffixe nominal) a une forme / $\mathrm{u} /$ (au lieu du /a/ kabyle, chleuh...), trait qui peut aussi être considéré comme caractéristique des parlers zénètes :

yis-u, «ce cheval », taddart-u, cette maison »; d'où les déictiques autonomes wu (w$u$ ), « celui-ci » et $t u(t-u)$, «celle-ci », en face de wa et $t a$, du kabyle, touareg, etc.

12 - Le suffixe personnel du nom (possessif) de $1^{\text {ère }}$ personne présente une forme, tout à fait spécifique, en - (i) $k^{w}$ (mon/ma/mes), en face du pan-berbère - $\mathrm{i} / \mathrm{y} / \mathrm{w}$ :

dad-i $-k^{w}$, « mon doigt »; imi- $k^{w}$, « ma bouche »; yewwa- $k^{w}$, « mon frère.

13 On pourrait y voir la trace d'une forme très anciennes (chamito-sémitique) de la marque de $1^{\text {ère }}$ personne (Chaker 2004), reconnaissable aussi dans le pronom autonome $n e k k(<n-k k)$, « moi ».

14 - Survivance de traces d'un thème d'aoriste intensif négatif (ou inaccompli négatif), trait lui aussi largement répandu dans le groupe zénète (Kossman 1989); en mozabite, 
il semble qu'il s'agisse d'un phénomène tout à fait résiduel puisque ce thème verbal est lié à un contexte syntaxique et sémantique bien précis: après négation, lorsque le groupe à valeur de futur :

tzara, «précéder habituellement » (aoriste intensif de zar) > tziri, dans le contexte wel tzirin, «ils ne précéderont pas » (en opposition à wel tzaran, «ils ne précèdent pas habituellement).

- Vocalisation en $/ \mathrm{u} /$ de la $3^{\mathrm{e}}$ et personne du singulier (il/elle) et de la $1^{\text {ère }}$ du pluriel (nous) au prétérit (accompli) des verbes à alternance finale $(\varnothing / \mathrm{i}-\mathrm{u})$, en face du /a/ kabyle, chleuh ou touareg :

yežžu, «il a laissé »; yenชu, «il a tué » (cf. kabyle, touareg, chleuh : yeğğa/yoyya, yenza/inza)...

16 - Parmi les morphèmes satellites du verbe, on constate que le mozabite ne connaît qu'une seule particule d'orientation spatiale, $d$ (orientation vers le locuteur) et semble totalement ignorer son pendant $n$ (éloignement/orientation vers l'auditeur). Cette configuration n'est cependant pas spécifique et on a noté depuis longtemps (A. Basset, Galand...) une nette tendance à la régression de la particule $n$, dans de nombreux parlers, y compris au sein de dialectes comme le kabyle qui connaissent les deux morphèmes.

17 - Présence et usage très fréquent, comme dans tous les parlers berbères de la bande méditerranéenne, de l'auxiliaire de prédication nominale $d$ (+ Nom à l'état libre) :

d něši i d ayyul mmi d šečč ?, "c'est moi qui suis un âne ou c'est toi ?»; netta d arğaz, « lui, c'est un homme », $d$ tametțut [t-tametțut], « c'est une femme ».

\section{Lexique}

18 Six petits glossaires mozabites ont été réalisés au XIX ${ }^{\mathrm{e}}$ siècle $:$ le vocabulaire de Shaler en 1830 qui reprend des matériaux recueillis par J. F. Schultze, celui de Samuda en 1840, celui de Hodgson en 1844, celui de Duveyrier en 1858, et enfin celui de Gourliau en 1898. Les cinq premiers sont repris dans R. Basset 1893 (p. 240 à 271).

19 Le travail le plus complet et le plus sûr en matière de lexicographie est évidemment le Ağraw n yiwalen tumzabt $t$-tfransist / Dictionnaire, mozabitefrançais, de J. Delheure (1984).

Un chercheur mozabite indépendant, A. Haouache a initié un important chantier collectif de constitution d'un dictionnaire général de la langue mozabite ; le projet, sur support informatique, est bien avancé.

Les terminologies de la littérature et de la linguistique, définies et mises en circulation dans les années 1970 dans les milieux militants kabyles, ont été toutes admises par les auteurs des manuels de mozabite (Abdesslam, Khalainou...). Cette première phase de " grammatisation » mozabite s'inscrit donc dans une dynamique pan-berbère.

Les mozabites recourent à un argot afin d'éviter qu'ils soient compris par les non berbérophones. En fait d'«argot», il s'agirait d'expressions et de périphrases. Un spécimen de cet argot a été recueilli par R. Basset en 1885, pendant son séjour au Mzab chez Motylinski. 


\section{Situation sociolinguistique}

23 Les Mozabites représentent une petite communauté qui ne compte que quelques centaines de milliers de locuteurs vivant dans un espace très resserré (vallée du Mzab), sous forme d'îlots isolés entourés d'une majorité de locuteurs arabophones. Les statistiques électorales ${ }^{1}$ (nombre de votants et résultats depuis 1989) permettent d'établir que les locuteurs du mozabite représentent un peu moins de $50 \%$ de la population de la wilaya de Ghardaïa, La grande partie d'entre eux se concentre dans la vallée du Mzab (les cinq cités). En 2005, la population totale de la wilaya de Ghardaïa était de 361.137 habitants : en extrapolant avec un taux de croissance moyen de 2,5\%, pour 2009, on obtiendrait $397.372 \mathrm{~h}$., ce qui ferait environ 200.000 locuteurs du mozabite. Mais il existe une importante diaspora mozabite dans les grandes villes $d u$ nord de l'Algérie, notamment à Alger, parmi laquelle l'usage familial et communautaire de la langue berbère reste la règle. Il est donc difficile d'être précis quant au nombre réel de locuteurs de ce dialecte et le chiffre de 200.000 doit certainement être considéré comme un minimum.

Grâce à son relatif isolement géographique et à sa spécificité religieuse, la communauté mozabite a pu résister à l'arabisation linguistique, bien qu'elle cohabite avec des groupes arabophones installés au Mzab depuis des siècles. Aujourd'hui, les spécificités religieuse et linguistique sont étroitement liées, au point qu'on ne peut guère imaginer un mozabite non ibadite et un ibadite non berbérophone. L'identité mozabite est donc fondée sur l'association étroite des deux éléments. Aussi, les cas de familles mozabites arabisées au niveau de leur pratique linguistique quotidienne sont-ils tout à fait exceptionnels.

On doit noter que la communauté mozabite mène un combat, toujours difficile malgré l'intégration ancienne de ses élites au projet national algérien: celui de la reconnaissance de sa doctrine ibadite, longtemps considérée comme " hérétique " par les théologiens sunnites orthodoxes, et même encore largement par l'ensemble de ses concitoyens, aussi bien arabophones que berbérophones. L'exclusion de l'ibadisme de la catégorie des rites considérés comme "voies justes ", ancrée à travers les siècles dans la culture religieuse sunnite, a laissé des blessures profondes dans la conscience collective des Mozabites et détermine prioritairement leur sentiment d'appartenance communautaire. Globalement, le religieux passe donc avant la langue.

L'arabe classique jouit évidemment du statut de langue du savoir religieux, détenu par une élite arabisante, qui détient aussi le pouvoir traditionnel. La langue arabe est enseignée de manière exclusive dans les écoles religieuses communautaires à tous les enfants mozabites, dès leur plus jeune âge.

En dehors d'un enseignement limité, portant sur quelques aspects de la littérature mozabite, dispensé depuis les années 1970 à l'Institut religieux El-Ișlah de Ghardaïa, le dialecte mozabite n'a commencé à être enseigné officiellement à l'école qu'à partir de l'année scolaire 1995-1996, suite au décret de mai 1995 créant le Haut Commissariat à l'Amazighité (HCA) et à l'instauration subséquente d'un enseignement facultatif de langue berbère au collège et au lycée. Dès cette rentrée 1995/96, 21 enseignants de ce dialecte ont été formés dans le cadre des stages organisé par le HCA. La moitié d'entre eux enseigneront cette première année à 584 élèves. Cependant, cet enseignement a rencontré de sévères difficultés d'ordre administratif, politique et pédagogique: blocage du recrutement des enseignants, hostilité de certains fonctionnaires de la 
Direction de l'Education de Ghardaïa, absence de supports pédagogiques, horaires inadéquats... Cet enseignement disparaîtra à partir de l'année 1999/2000 et, ce n'est qu'en 2003/2004, qu'un unique enseignant est autorisé à dispenser ce cours pour 76 élèves de l'enseignement moyen (collège). Cette expérience apparait donc, au moins pour l'instant, comme un échec très net: selon les données officielles du MEN et du HCA, pendant l'année 2008/2009, l'enseignement de tamazight, assuré par un seul enseignant, n'était plus proposé qu'au sein de deux établissements sur les 224 (tous cycles confondus) que compte le département de Ghardaïa et il ne touche que 19 élèves !.

Un cours de tumzababt est créé en 1990 au Département de Langue et Culture Amazighes de l'Université de Tizi-Ouzou et est toujours assuré.

Influencé par le «Printemps berbère » en Kabylie, la revendication identitaire s'est développée au Mzab parmi les jeunes intellectuels à partir de début des années 1980 ; les idées berbéristes se transmettaient notamment dans le milieu universitaire. On a assisté ainsi au Mzab à un éveil culturel remarquable qui se manifeste par la création de chorales de chants mozabites. Ces troupes qui animent des fêtes de mariage partout dans les villes du Mzab, ont réussi à ressusciter des chants berbères anciens, et à les sauver ainsi de l'oubli. La conscience identitaire berbère dans la jeunesse mozabite s'est accentuée avec l'avènement du pluralisme politique en 1989, et avec les activités du mouvement culturel berbère, qui tendait, à la fin des années 1980, à donner une dimension nationale à la revendication amazighe.

Néanmoins l'élite mozabite arabisante, détentrice des savoirs anciens exclusivement véhiculés par l'arabe littéraire, et détenant à ce titre le pouvoir et la légitimité traditionnels, manifeste une grande indifférence voire, parfois, une hostilité marquée envers la revendication amazighe portée essentiellement par des jeunes universitaires de culture et de formation modernes.

\section{Littérature}

31 La culture arabe de l'élite religieuse du Mzab a longtemps primé sur la littérature de langue berbère, restée orale et nettement dévalorisée. Ceci explique l'absence quasitotale d'une tradition poétique mozabite ancienne, incarnée par des auteurs reconnus, contrairement à la situation qui prévaut en Kabylie. Alors que les Mozabites disposent d'une littérature religieuse abondante en langue arabe, et ce depuis Al-Wargalani (XII ${ }^{\mathrm{e}}$ ) la littérature mozabite de langue berbère est restée jusqu'à une date très récente de caractère exclusivement oral, ce qui a, en partie, favorisé sa disparition.

Et ce n'est qu'au du XX⿳亠丷厂 ${ }^{e}$ siècle, qu'un éveil culturel au sein des jeunes générations a commencé à ressusciter ce qui restait de cette littérature berbère, parallèlement à un passage à l'écrit, utilisant à la fois les caractères latins et arabes.

33 Cependant, plusieurs textes religieux, notamment l'Aqida (profession de foi, doctrine) ibadite ont été écrits en berbère. Ce texte doctrinal fondamental, que tout mozabite doit apprendre par cœur en signe du baptême, indique dès son début qu'il était originellement écrit en berbère. Selon Motylinski (1905 : 505), le Chikh Joumay l'aurait traduite en arabe "afin qu'elle soit facile à comprendre [...]».Malheureusement, aucune trace n'a été retrouvée du texte berbère originel, probablement parce qu'il aura été brûlé pour éviter toute interférence avec le texte arabe. 
Un autre manuscrit comportant 24 fragments en berbère a été repéré par T. Lewicki, dans la collection de Smogarzewski (1927). Il a été recueilli à Ghardaïa en 1913 par quatre scripteurs mozabites (Ould Braham 1987, p. 87, 88).

A l'instar de W. Vycichl qui considère l'existence d'un «argot » comme trait commun aux trois communautés ibadites berbères (Djebel Nefousa, Djerba, Mzab), V. Brugnatelli avance même la thèse de l'existence d'une koinè littéraire commune à ces trois communautés.

Dès le début du XXe siècle, des bribes de la littérature mozabite ont été transcrits dans le cadre d'études ethnographiques; A. Moulieras (1895) fut le premier à transcrire des fables et des contes mozabites. En 1918, P. Leblanc de Prébois, publia un ensemble de légendes mozabites. Des éléments de la littérature féminine ont été transcrits par Gouvion en 1926, ainsi que par Goichon en 1927. On peut craindre qu'une grande partie de cette littérature orale ait déjà disparue.

La période récente a vu l'apparition de jeunes poètes en langue mozabite tels que Fekhar, Tirichine,... et de chercheurs comme A. Haouache, grammairien et initiateur du grand dictionnaire mozabite, travail collectif désormais finalisé et qui devrait paraître prochainement (publications du HCA).

C'est généralement l'alphabet arabe qui était utilisé pour la notation des œuvres littéraires publiées, mais les œuvres littéraires (Tirichine 2007) et travaux les plus récents de codification de la langue recourent tous à l'alphabet latin (Abdesslam, Haouache...), même lorsque la langue de travail est l'arabe.

\section{BIBLIOGRAPHIE}

ABDESSLAM I. \& ABDESSLAM B., Précis d'écriture et de grammaire de la langue berbère (mozabite), [en arabe], Ghardaïa, 1996, $187 \mathrm{p}$.

AULARD, « La vie dans le Mzab. Textes bilingues (1938-1941 », Etudes et Documents Berbères, 5, 1989, p. 131-168.

BASSET R., 1887, Manuel de langue kabyle (dialecte zouaoua), Maisonneuve et Leclerc. Paris : un texte. BASSET R., Etude sur la zénatia du Mzab, de Ouargla et de l'Oued Rir', Paris, Leroux (Publ. Ecole des Lettres d'Alger - Bulletin de Correspondance Africaine), 1893.

BASSET R., Loqman berbère, Paris, Leroux, 1890 : 9 textes.

BASSET R., « Notes de lexicographie berbère », $4^{\mathrm{e}}$ série, Journal asiatique, 1877 et tiré-à-part, Paris, Imprimerie nationale, 1888 ( Argot du Mzab»). (réédité en Algérie par les Editions Guraya, 2008).

BEN SI LOUNIS A. N. \& BEN YEHIA M.,, Grammaire Mozabite, Alger, Imprimerie Baldachino-LarondeViguier, 1897.

BENTOLILA F. (Dir.), Devinettes berbères, Paris, CILF (Fleuve et Flamme, Textes bilingues), 1986 ; voir t. 2, chap. IX, p. 335-352 : « Devinettes du Mzab», par Bouhounali H. B. 
BIARNAY, Notes d'ethnographie et de linguistique nord-africaine, Paris, Leroux (IHEM) 1924 : p. 165-265, texte et glossaire.

BOUSSADA A., Ahewwef $n$ weylan [= Poésie du Mzab], Ghardaia, 2006.

BRUGNATELLI V., « Notes d'onomastique jerbienne et mozabite », in K. Naït-Zerrad, D. Ibriszimow, R. Vossen (éd.), Nouvelles études berbères.., Actes du $2^{\mathrm{e}}$ Bayreuth-Frankfurter Kolloquium zur Berberologie, Köln, Rüdiger Köppe Verlag (Berber Studies vol. 8), 2004, p. 125-141.

CHAKER S., "Les paradigmes personnels du berbère ", Systèmes de marques personnelles en Afrique (édité. par. D. Ibriszimow et G. Segerer), Louvain/Paris, Peeters (« Afrique et Langage »: 8), 2004, p. 43-54.

DALLET J.-M., Récits du Mzab, Fort National, Fichier de Documentation Berbère 61, 1961, réédité en hors série en 1965.

DALLET J.-M., Oued Mzab. Pour une vérification des notations berbères de E. GOURLIAU (1898), Fort National, Fichier de Documentation Berbère, 1969.

DALLET J.-M., Berbère de l'Oued Mzab : le verbe, glossaire, classification. Fort National, FDB, 1970.

DELHEURE J., [Contes] in DEJEUX J. Djoh'a, héros de la tradition orale arabo-berbère : hier et aujourd'hui, Montréal ; Sherbrooke, Naaman, 1978.

DELHEURE J. \& ALLAIN M., « Le travail de la laine à Ghardaïa », Revue de l'Occident Musulman et de la Méditerranée, 27, 1979, p. 6-74.

DELHEURE J., Ağraw n yiwalen tumzzabt $t$-tfransist / Dictionnaire, mozabite-Français, Paris, SELAF/

Peeters, 1984.

DELHEURE J., Fait et dires du Mzab/Iwalen d tmeğğga n At Mzạb, Paris, SELAF/Peeters, 1985.

DELHEURE J., Etude sur la langue mozabite, Paris, Pères Blancs, 1986, 57 p. multigraphiées ; publiée sous forme définitive sous le titre : « Etude sur le mozabite », Etudes et Documents berbères, 6 ; 1989, p. 20-157.

DELHEURE J., « Systèmes verbaux ouargli-mozabites », Journées d'étude de linguistique berbère, Paris, Publications Langues'O, 1989, p. 53-64.

DELHEURE J., « Calendrier agraire de Ouargla (et Mzab) », Encyclopédie Berbère, 11, 1992, p. 1717-1719.

FEKHAR A. H., Imețțawen n lferḥ [= Larmes de joie], Ghardaïa, 1985. (Recueil de poésies).

FEKHAR A. H, [Deux poèmes], Awal, 2, 1986, 191-196.

FEKHAR A. H., Imețtawen izeggayen [= Larmes rouges], Ghardaïa, 1990. (Recueil de poésies).

FEKHAR A. H., Taydart $n$ Weylan [= l'Epi du Mzab], Ghardaïa, 1994. (Théâtre). Fichier de Documentation berbère : publication régulière de textes mozabites, cf. Le Fichier périodique, 124, 1974 (IV), Table chronologique et index, par J. Lanfry, p. 71, « Beni Mzab »; Voir aussi le fascicule 126, 1975 (II), « L'hydraulique traditionnelle à Ouargla et au Mzab ».

Gorchon A. M., La vie féminine au Mzab, étude de sociologie musulmane, Paris, Paul Geuthner, 1927. GOURLIAU E., Grammaire complète de la langue mozabite, comparée dans ses parties essentielles aux dialectes kabyle et tamachek', Miliana, Imprimerie Legendre, 1898.

GoUvion M. \& E., Le kharedjisme, monographie du Mzab, 1926, Casablanca.

HANOTEAU A., Grammaire kabyle, Alger, A. Jourdan, (1858), 1906 (2e édition) : un texte, p. 357. 
HANOTEAU A., Lettre à Reinaud [sur « le système de numération chez la race berbère], Journal asiatique, 16, 1860, p. 264-269.

HAOUACHE A et al., « De quelques créations lexicales à partir des racines berbères en usage dans le Sud algérien ", Awal, 11, 1994, p. 137-148.

IGHOULAD B., « Deux textes de l'oued Mzab », Etudes et Documents Berbères, 1, 1986, p. 64-67.

KHALAINOU M. Tajerrumt $n$ tmaziðt (tumzabt), Beni Izguen, 2006.

LEBLANC de PREBois P., «Légendes du Mzab », Bulletin de la Société de Géographie d'Alger, 23, 1918, p. 93-115.

LEWICKI T., "Quelques textes inédits en vieux berbère provenant d'une chronique ibadite anonyme », Revue des Etudes Islamiques, 1934/3. p. 275-305. [Textes datant du XII ${ }^{e}$; suivi d'une « Note additionnelle » d'A. Basset p. 297-305.]

KOSSMANN M., «L'inaccompli négatif en berbère », Etudes et Documents Berbères, 6, 1989, p. 19-29.

KOSSMANN M., " La conjugaison des verbes CC à voyelle alternante en berbère ", Etudes et

Documents Berbères, 12, 1995, p. 17-33

KosSmann M., "Les verbes à finale $i$ en zénète : étude historique », Etudes et Documents Berbères, 13, 1995, p. 99-104

KoSSMANN M., Essai sur la phonologie du proto-berbère, Köln, Rüdiger Köppe Verlag, 1999.

MAMMERI M., C. R. de «Imețtawen n lferḥ de FEKHAR A. H. », Awal, 1, 1985, p. 194-196.

MASQUERAY E., Comparaison d'un vocabulaire du dialecte des zenaga du Sénégal avec les vocabulaires correspondants des dialectes des Chawia et des Beni Mzab, Paris, Imprimerie Nationale, 1879 (extrait des «Archives des missions scientifiques et littéraires », $3^{\mathrm{e}}$ série ; 1878).

MOTYLINSKI-CALASSANTI A. (de), «Le nom de Dieu chez les Berbères », Revue Africaine, Alger, 1905, p. 141-148. [Texte datant du XIII'].

MOTYLINSKI-CALASSANTI A. (de), «L'Aqida des Abdhites ", in Recueil de mémoires et de textes publiés en l'honneur du XIVe congrès des orientalistes, par les professeurs de l'Ecole supérieur d'Alger, Alger, 1905.

MOULIERAS A., Les Béni-Isguen (Mzab), essai sur leur dialecte, Oran, Fouque, 1895.

NAÏT-ZERRAD K., « Esquisse d'une classification linguistique des parlers berbères », Al-AndalusMagreb, VIII-IX/2, 2002, p. 389-412.

NOUH A., Le vocabulaire berbère commun au kabyle et au mozabite, étude de lexicologie berbère comparée, Mémoire de Magister, Université Mouloud Mammeri (Tizi-Ouzou), 2008.

NOUH A., Glossaire du vocabulaire commun au kabyle et au mozabite, HCA, Alger, 2008.

OULD BRAHAM O., « Lecture des 24 textes berbères médiévaux extraits d'une chronique Ibadite par T. Lewicki », Littérature Orale Arabo-Berbère, n 18, 1987, p. 87-125

OULD BRAHAM O., « Emile Masqueray au Mzab. A la recherche des livres ibâdites », Etudes Documents Berbères, 9, 1993, p. 5-35.

SAMUDA, (pseud.), « Essai sur la langue des Beni Mzab », Le moniteur algérien, 1840 [mars, avril].

TIRICHINE S., Ulinu, [= Mon cœur], Ghardaïa, Dâr el-Kotb, 1996, 325 p. 2e édition, en caractères latins. Alger, ENAG, 2007 [recueil de poésies]. 
VYCICHL W. « Argot (Jebel Nefousa-Jerba-Mzab) », Encyclopédie berbère, 6, 1989, p. 882-884.

WILLMS Alfred, Die dialektale Differenzierung des Berberischen, Berlin, Reimer (Afrika und Übersee), 1980.

\section{Autres documents}

Ministère de l'Education Nationale (Algérie), Inspection générale. Enseignement de Tamazight. Etats des lieux. Année scolaire 2006/2007. 14 p. [rapport interne de l'inspection générale des enseignements].

Le pays / Tamurt, hebdomadaire régional de Kabylie $(1991 \rightarrow 1995)$. Voir notamment :

Salah Tirichine (entretien avec-) « Faire fleurir nos racines amazighes », $n^{\circ} 30$, daté du 30/11/1991, p. 17.

Adel Mzab (entretien avec-) « On doit évoluer partout ». nº 32, daté du 14/12/1991, p. 17.

\section{NOTES}

1. Cf. notamment Loi 89-15 du 22/08/1989 fixant les circonscriptions électorales et le nombre de siège à pourvoir pour le renouvellement des assemblés populaires communales et des assemblées populaires de wilaya.

INDEX

Mots-clés : Algérie, Dialectologie, Langue, Mzab, Poésie 